\title{
Bases of variational method for calculating of metal-cutting systems accuracy
}

\author{
Olesya Anikeeva ${ }^{1, *}$, Alexander Ivakhnenko ${ }^{1}$, and Oleg Erenkov ${ }^{2}$ \\ ${ }^{1}$ South-West State University, 305040 Kursk, Russia \\ ${ }^{2}$ Pacific National University, 680035 Khabarovsk, Russia
}

\begin{abstract}
The bases of a variation method to calculate the metal-cutting systems accuracy for the first time are systemically stated in this paper. The main attention is paid to a problem of joint influence of geometrical accuracy of machine tools and cutting tools parameters on the details processed surfaces accuracy. The general mathematical model of metalcutting systems accuracy is presented in this work. The general model is the basis to develop the full mathematical model of turning metal-cutting system accuracy. The results of accuracy modeling at turning with a wide cutter are received when studying nature of influence of the lathe and the cutting tool geometrical errors on processing accuracy of cylindrical and face surfaces. The directions of further researches in the field of calculations of metal-cutting systems accuracy at difficult surfaces processing by the shaped cutting tool and also when accounting rigidity of the systems elements and the proceeding processes are revealed in the presented work.
\end{abstract}

\section{Introduction}

The geometrical accuracy one of the main indicators for characterizing both the processing and the equipments and tools used. The basis of this work is the variation method for metalcutting machines accuracy calculation $[1,2]$ which is using uniform coordinates and gained development in the next works [3-5]. At processing accuracy researches on metal-cutting machines the main attention is paid to the accuracy of the machine tools though the structure of the metal-cutting system (MCS) always includes the cutting tool. In this work the main attention is paid to a problem of joint influence of machines and the cutting tools geometrical accuracy parameters on the accuracy of the details treated surfaces.

\section{General mathematical model of metal-cutting systems accuracy}

The equation of the nominal processed surface has the appearance:

$$
\boldsymbol{r}_{\mathbf{0}}=\boldsymbol{r}_{\mathbf{0}}\left(u, v, \boldsymbol{q}_{\mathbf{0}}\right)
$$

* Corresponding author: olesya-anikeeva@ yandex.ru 
where $u, v$ - curvilinear coordinates of a surface; $\boldsymbol{q}_{\mathbf{0}}$ - a vector of surface dimensional parameters.

The Form-Shaping Function (FSF) characterizing structure of MCS has the appearance:

$$
\boldsymbol{r}_{0}=\boldsymbol{A}_{c m} \boldsymbol{r}_{p u},
$$

where $\boldsymbol{r}_{\mathbf{0}}$ - the radius vector connecting of the cutting tool coordinates points in the cutting tool coordinates systems and the blank being processed; $\boldsymbol{A}_{\boldsymbol{c m}}$ - the matrix of the machine coordinates transformations which is the work of the known matrixes of elementary movements $[1,2] ; \boldsymbol{r}_{\boldsymbol{p}}$ - the radius vector of the tool form-building points.

Except imposing on the Form-Shaping Function of the known bonds such as rounding, hidden and functional it is also necessary to consider the cutting tool parameters and then FSF in expression (1) will correspond to the nominal processed surface equation:

$$
\boldsymbol{r}_{\mathbf{0}}=\boldsymbol{r}_{\mathbf{0}}\left(u, v, \boldsymbol{q}_{\mathbf{1}}, \boldsymbol{q}_{2}\right),
$$

where $\boldsymbol{q}_{\mathbf{1}}$ - the vector of the machine bonds; $\boldsymbol{q}_{\mathbf{2}}$ - the dimensional bonds vector of the tool cutting edge.

The functional purpose of the MCS elements is implemented at equality of expressions (1) and (3), that is:

$$
\boldsymbol{r}_{\mathbf{0}}\left(u, v, \boldsymbol{q}_{\mathbf{0}}\right)=\boldsymbol{r}_{\mathbf{0}}\left(u, v, \boldsymbol{q}_{1}, \boldsymbol{q}_{2}\right) .
$$

Using the approach stated in [6] we will define a full variation of expression (2):

$$
\Delta r_{0}=\Delta A_{c m} r_{p u}+A_{c m} \Delta r_{p u}+\Delta A_{c m} \Delta r_{p u} .
$$

Expression (5) is the first main dependence for creation of a full (nonlinear) variation method for calculation of MCS accuracy. It is visible from the expression structure that vector errors budget $-\Delta \boldsymbol{r}_{0}$ of MCS contains the nonlinear member $-\Delta \boldsymbol{A}_{\boldsymbol{c} m} \Delta \boldsymbol{r}_{\boldsymbol{p} u}$. The principal difference from known [2] nonlinear problems of machines accuracy consists in account as structurally nonlinear member $\Delta \boldsymbol{A}_{\boldsymbol{c}} \Delta \boldsymbol{r}_{p u}$, and also in reasonable consideration of sizes of the second and subsequent orders of smallness in $\boldsymbol{A}_{\boldsymbol{c} m} \Delta \boldsymbol{r}_{p u}$, component that is the direction of separate researches.

The linearized representation of full variation of expression (2) has the following appearance:

$$
\Delta r_{0}=\Delta A_{c m} r_{p u}+A_{c m} \Delta r_{p u},
$$

and is also the main dependence of linear variation method for calculation of MCS accuracy as includes the vector errors budget $-\Delta \boldsymbol{r}_{0}$ machine errors $\left(\Delta \boldsymbol{A}_{\boldsymbol{c} m}\right)$ and of the cutting tool errors $\Delta \boldsymbol{r}_{\boldsymbol{p} u}$. In accordance with the variation method for calculation of metal-cutting machines accuracy $[1,2]$ believe the cutting tool is ideal or the tool is point wise $\boldsymbol{r}_{p u}=(0 ; 0 ; 0 ; 1)^{T}$ that allows to consider $\Delta \boldsymbol{r}_{p u}=0$.

The first order of smallness members only are fixed in the expression (6) of this work at variations $\Delta \boldsymbol{A}_{\boldsymbol{c} m}$ and $\Delta \boldsymbol{r}_{\boldsymbol{p} u}$ consideration.

The second main dependence of a variation method for calculation of MCS accuracy both in nonlinear and in linear statement is the corresponding variation of expression (4):

$$
\Delta \boldsymbol{r}_{0}\left(u, v, \Delta q_{0}\right)=\Delta \boldsymbol{r}_{0}\left(u, v, \Delta q_{1}, \Delta q_{2}\right) .
$$

The calculation rules of variations in expression (7) don't differ from the corresponding rules used in works $[1,2]$. 


\section{Mathematical model of turning metal-cutting system accuracy}

The significant share among different types of processing is occupied by well study turning which is a test example for the results received by different authors [1, 2, 4, 7-9].

Form-Shaping Function (2) of lathes has an appearance:

$$
r_{0}=A^{6}(\varphi) A^{3}(z) A^{1}(x) r_{p u}
$$

where $\boldsymbol{A}^{\mathbf{6}}(\varphi)$ - turn matrix around axis $Z ; \boldsymbol{A}^{\mathbf{3}}(z)$ and $\boldsymbol{A}^{\mathbf{1}}(x)$ - matrixes of movements along axes $Z$ and $X$, respectively; $\boldsymbol{r}_{p u}=\left(x_{p u} ; y_{p u} ; z_{p u} ; 1\right)^{\mathrm{T}}-$ the radius vector of the cutting cutter edge. After calculations from expression (8) we will receive:

$$
\boldsymbol{r}_{\mathbf{0}}=\left(\begin{array}{c}
\left(x+x_{p u}\right) \cos \varphi-y_{p u} \sin \varphi \\
\left(x+x_{p u}\right) \sin \varphi+y_{p u} \cos \varphi \\
z+z_{p u} \\
1
\end{array}\right)
$$

The vector errors budget of metal-cutting system is equal:

$$
\Delta r_{0}=\left(\varepsilon_{0} A^{6} A^{3} A^{1}+A^{6} \varepsilon_{1} A^{3} A^{1}+A^{6} A^{3} \varepsilon_{2} A^{1}+A^{6} A^{3} A^{1} \varepsilon_{3}\right) r_{p u}+A^{6} A^{3} A^{1} \Delta r_{p u}
$$

where $\Delta \boldsymbol{r}_{\boldsymbol{p} u}=\left(\Delta x_{p u} ; \Delta y_{p u} ; \Delta z_{p u} ; 0\right)^{\mathrm{T}}-$ variation of the radius vector of the cutting cutter edge; $\boldsymbol{\varepsilon}_{i}$ - matrixes of elementary errors $(i=0, \ldots, 3)$ :

$$
\boldsymbol{\varepsilon}_{i}=\left(\begin{array}{cccc}
0 & -\gamma_{i} & \beta_{i} & \delta x_{i} \\
\gamma_{i} & 0 & -\alpha_{i} & \delta y_{i} \\
-\beta_{i} & \alpha_{i} & 0 & \delta z_{i} \\
0 & 0 & 0 & 0
\end{array}\right)
$$

After substitution of the corresponding expressions for matrixes: $A^{\mathbf{6}}, \boldsymbol{A}^{\mathbf{3}}, \boldsymbol{A}^{\mathbf{1}}$ and vectors: $\boldsymbol{r}_{\boldsymbol{p}}$ and $\Delta \boldsymbol{r}_{\boldsymbol{p}}$ in expression (10) we will receive:

$$
\Delta \boldsymbol{r}_{\mathbf{0}}=\left(\begin{array}{c}
\Delta_{1} \cos \varphi-\Delta_{2} \sin \varphi+\Delta_{3} \\
\Delta_{1} \sin \varphi+\Delta_{2} \cos \varphi+\Delta_{4} \\
\Delta_{5} \\
0
\end{array}\right)
$$

where $\Delta_{1}=\sum_{i=1}^{3} \delta x_{i}+z \beta_{1}+z_{p u} \sum_{i=1}^{3} \beta_{i}-y_{p u} \sum_{i=0}^{3} \gamma_{i}+\Delta x_{p u}$;

$$
\begin{aligned}
& \Delta_{2}=\sum_{i=1}^{3} \delta y_{i}-z \alpha_{1}-z_{p u} \sum_{i=1}^{3} \alpha_{i}-x_{p u} \sum_{i=0}^{3} \gamma_{i}+\Delta y_{p u} ; \Delta_{3}=\delta x_{0}+\beta_{0}\left(z+z_{p u}\right) ; \\
& \Delta_{4}=\delta y_{0}-\alpha_{0}\left(z+z_{p u}\right) ; \\
& \Delta_{5}=\left(\alpha_{0} \sin \varphi-\beta_{0} \cos \varphi\right)\left(x+x_{p u}\right)+\left(\alpha_{0} \cos \varphi-\beta_{0} \sin \varphi\right) y_{p u}-x \sum_{i=1}^{2} \beta_{i}-x_{p u} \sum_{i=1}^{3} \beta_{i}+y_{p u} \sum_{i=1}^{3} \alpha_{i}+ \\
& +\sum_{i=0}^{3} \delta z_{i}+\Delta z_{p u} .
\end{aligned}
$$


We will present a single vector as a normal to any processed surface by cutting tool in turning metal-cutting system in the form of $\boldsymbol{n}=\left(n_{x} ; n_{y} ; n_{z} ; 0\right)^{T}$. Scalar errors budget $\Delta r_{n}$ with accuracy components $\Delta_{1}, \Delta_{2}, \Delta_{3}, \Delta_{4}$ and $\Delta_{5}$ coincides with the budget presented in [1,2]:

$$
\Delta r_{n}=\Delta_{1}\left(n_{x} \cos \varphi+n_{y} \sin \varphi\right)+\Delta_{2}\left(n_{x} \sin \varphi+n_{y} \cos \varphi\right)+\Delta_{3} n_{x}+\Delta_{4} n_{y}+\Delta_{5} n_{z} .
$$

Expressions $(8) \div(12)$ are full mathematical model of turning metal-cutting system accuracy.

\section{Modeling of accuracy at turning by a wide cutter}

We will consider of cylindrical and face surfaces with a wide cutter.

When processing a cylindrical surface with a radius of $R$ is processed the equation of communications for matrixes of $\boldsymbol{A}^{\mathbf{1}}(x)$ and $\boldsymbol{A}^{\mathbf{3}}(z)$ has an appearance: $x=R ; z=0$, and the equation of the cutting edge $\boldsymbol{r}_{\boldsymbol{p}}=(0 ; 0 ; z ; 1)^{T}$. The equation of a nominal surface $\boldsymbol{r}_{0}=(R \cdot \cos \varphi ; R \cdot \sin \varphi ; \quad z ; 1)^{T}$, a single vector of a normal to this surface $\boldsymbol{n}=(\cos \varphi ; \sin \varphi ; 0 ; 0)^{T}$.

We will define scalar errors budget using a scalar product of vectors $\Delta r_{n}=\Delta \boldsymbol{r}_{\mathbf{0}} \cdot \boldsymbol{n}$ :

$$
\Delta r_{n}=\sum_{i=1}^{3} \delta x_{i}+z \sum_{i=1}^{3} \beta_{i}+\Delta x_{p u}+\left(\delta x_{0}+\beta_{0} z\right) \cos \varphi+\left(\delta y_{0}-\alpha_{0} z\right) \sin \varphi
$$

The scalar errors budget $\Delta r_{n}$ at a cylindrical surface turning with a dot cutter $[1,2]$ doesn't include an error of the cutting tool edge $\Delta x_{p u}$, and it contains only one component $\beta_{1}$ instead of the sum $\sum_{i=1}^{3} \beta_{i}$.

Thus the productivity increase reached when processing by a wide cutter potentially has the smaller accuracy as $\Delta r_{n}$ contains in expression (13) three components more than when processing by a dot cutter.

Taking into account (13), the equation of radius vector of the real processed surface with use of new determination of vector errors budget $\Delta \boldsymbol{r}_{0}{ }^{* *}$ [4] has an appearance:

$$
r=\left(\begin{array}{c}
R \cos \varphi+\Delta_{1}^{* *} \cos \varphi+\Delta_{3}^{* *} \\
R \sin \varphi+\Delta_{1}^{* * *} \sin \varphi+\Delta_{4}^{* *} \\
z+\Delta_{5}^{* *} \\
1
\end{array}\right),
$$

where $\Delta_{1}^{* *}=\sum_{i=1}^{3} \delta x_{i}+z \sum_{i=1}^{3} \beta_{i}+\Delta x_{p u} ; \Delta_{3}^{* *}=\delta x_{0}+z \beta_{0} ; \Delta_{4}^{* *}=\delta y_{0}-z \alpha_{0}$

$\Delta_{5}^{* *}=R\left(\alpha_{0} \sin \varphi-\beta_{0} \cos \varphi-\sum_{i=1}^{2} \beta_{i}\right)$.

The equation of the real processed surface radius vector has included only geometrical errors entering in expression (13). Influence of the majority of the machine elementary geometrical errors entering in expression (13) is rather well studied $[1,2,4,7]$. Here we will consider influence of machine elementary geometrical errors $\left\{\beta_{2}, \beta_{3}\right\}$ and cutting tool edge errors $\Delta x_{p u}$ on processing accuracy. We will consider that $\beta_{2}=$ const, $\beta_{3}=$ const $\left(\beta_{2} \neq 0 ; \beta_{3} \neq 0\right)$, making variations of radius vector of the cutting cutter edge $\Delta x_{p u}=d x_{p u}+\delta x_{p u}$, and $d x_{p u}=$ const, and the private variation $\delta x_{p u}$ is equal to some function from a variable $z$, i.e. $\delta x_{p u}=\delta x_{p u}(z)$. The orthogonal polynoms of Fourier, Hermite and 
others with final quantity of components or different types of splines $[1,2,10]$ are used for private variations representation. Here we use representation $\delta x_{p u}=\delta_{1} \sin (\pi z / L)$, where $\delta_{1}<<1 ; L-$ cutter width.

In fig. 1 influence of MCS geometrical errors on size and form errors at cylindrical surface processing is shown.

a

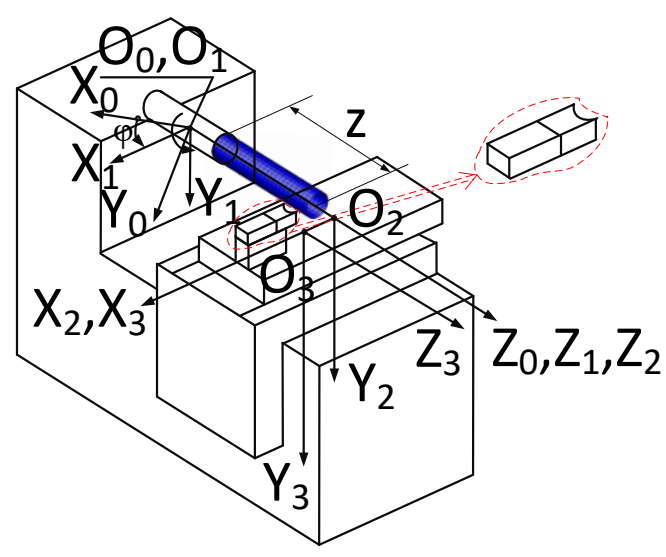

c

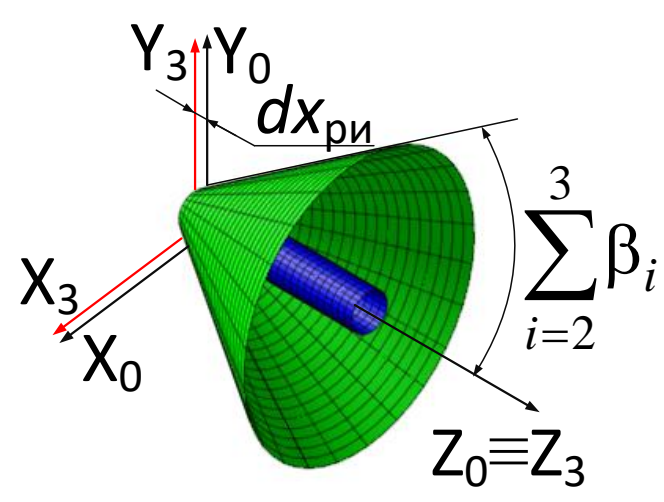

b

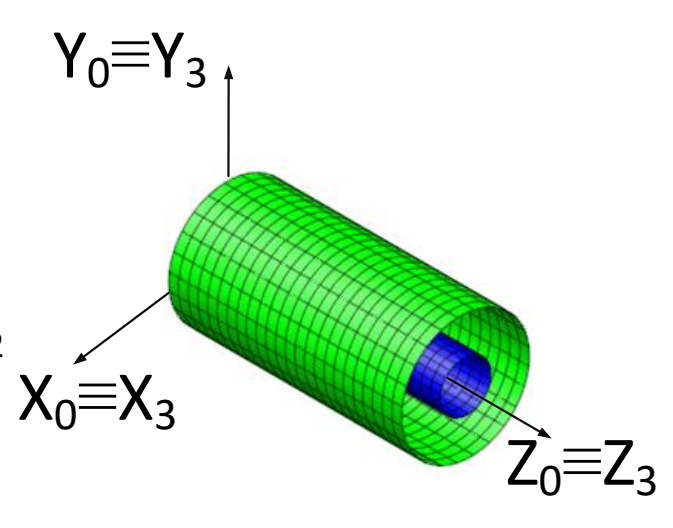

$\mathrm{d}$

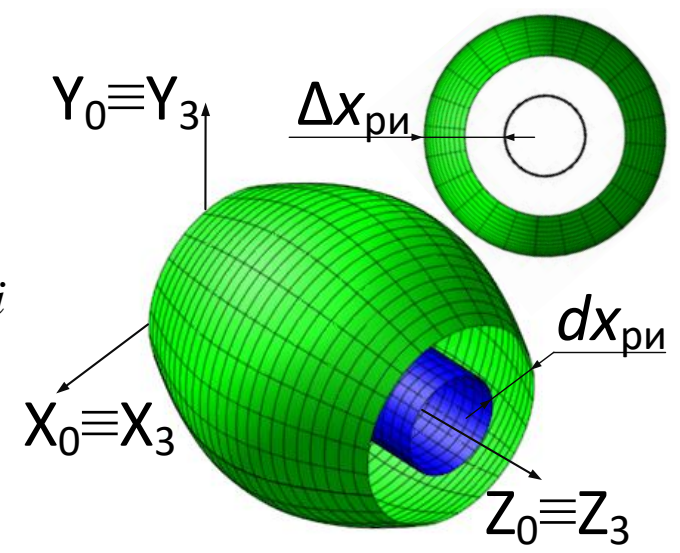

Fig. 1. Influence of MCS geometrical errors on errors of cylindrical surface processing: a) the formbuilding system of the machine and the nominal processed surface (cylinder); b) formation of the real surface size error $d x_{p u} \neq 0$; c) formation of a real surface form error at $\beta_{2} \neq 0 ; \beta_{3} \neq 0$; d) formation of a real surface form error at $\delta x_{p u} \neq 0$.

The nature of influence of the machine and the cutting tool geometrical errors on the cylindrical surface processing accuracy is visible from fig. 1:

- The constant component of variation $d x_{p u}$ exerts impact on formation of the real surface size error,

- Errors $\beta_{2}$ and $\beta_{3}$ exert impact on formation of the form error - conicity of the processed surface,

-The variable component of variation $\delta x_{p u}(z)$ is copied on the processed surface. 
In case of the face surface processing with radius of $R$ the equation of communications for matrixes of $\boldsymbol{A}^{\mathbf{1}}(x)$ and $\boldsymbol{A}^{\mathbf{3}}(z)$ have an appearance: $x=0 ; z=c$, and the equation of the cutting edge $\boldsymbol{r}_{\boldsymbol{p} u}=(x ; 0 ; 0 ; 1)^{T}$. The equation of the nominal surface $\boldsymbol{r}_{\mathbf{0}}=(x \cdot \cos \varphi ; x \cdot \sin \varphi ; c ; 1)^{T}$, the single vector of the normal to this surface $-\boldsymbol{n}=(0 ; 0 ; 1 ; 0)^{T}$.

The scalar errors budget is equal:

$$
\Delta r_{n}=x\left(\alpha_{0} \sin \varphi-\beta_{0} \cos \varphi\right)-x \sum_{i=1}^{3} \beta_{i}+\sum_{i=0}^{3} \delta z_{i}+\Delta z_{p u} .
$$

The scalar errors budget $\Delta r_{n}$ at the face surface processing with dot cutter [1,2] doesn't include the cutting edge error of the tool $\Delta z_{p u}$ and instead of the sum $\sum_{i=1}^{3} \beta_{i}$ it contains the sum $\left(\beta_{1}+\beta_{2}\right)$. This scheme of processing has potentially the smaller accuracy as $\Delta r_{n}$ contains in expression (15) two components more than at processing by the dot cutter.

Taking into account (15) the equation of the real processed surface radius vector has an appearance:

$$
\boldsymbol{r}=\left(\begin{array}{c}
\Delta_{1}^{* *} \cos \varphi+\Delta_{3}^{* *} \\
\Delta_{1}^{* *} \sin \varphi+\Delta_{4}^{* *} \\
c+\Delta_{5}^{* *} \\
0
\end{array}\right),
$$

where $\Delta_{1}^{* *}=c \sum_{i=1}^{3} \beta_{i} ; \Delta_{3}^{* *}=c \beta_{0} ; \Delta_{4}^{* *}=-c \alpha_{0} ; \Delta_{5}^{* *}=x\left(\alpha_{0} \sin \varphi-\beta_{0} \cos \varphi\right)-x \sum_{i=1}^{3} \beta_{i}+\sum_{i=0}^{3} \delta z_{i}+\Delta z_{p u}$.

We will consider influence of the machine elementary geometrical error $\beta_{3}$ and of the cutting edge error of the tool $\Delta z_{p u}$ on processing accuracy. Still, $\beta_{3}=$ const $\left(\beta_{3} \neq 0\right)$ making variations $\Delta z_{p u}=d z_{p u}+\delta z_{p u}, d z_{p u}=$ const, $\delta z_{p u}=\delta_{2} \sin (\pi x / R)$, where $\delta_{2} \ll<1$.

In fig. 2 influence of MCS geometrical errors on the size and form errors at a face surface processing is shown.

The nature of influence of the machine and the cutting tool geometrical errors on the face surface processing accuracy is visible from fig. 2 :

- The constant component of variation $d z_{p u}$ exerts impact on formation of the real surface size error,

- Errors $\beta_{3}$ exert impact on formation of the form error - conicity of the processed surface,

- The variable component of variation $\delta z_{p u}(x)$ as well as $\delta x_{p u}(z)$ is copied on the processed surface.

So the nature of the lathe and the cutting tool geometrical errors influence on the accuracy of processing of cylindrical and face surfaces is considered in this section.

\section{The direction of further researches in the field of the metal- cutting systems accuracy}

The given above calculations of processing accuracy in metal-cutting systems don't consider influence of rigidity of these systems elements and also a temporary factor on change of their parameters. The further researches direction in the field of the metal-cutting systems accuracy is connected to taking into account static and temperature deformations and also fluctuations and wear of the cutting tool, machine units and their joints. Also the tasks connected with accuracy calculations when using of the shaped cutting tool for processing of difficult surfaces have considerable interest. 

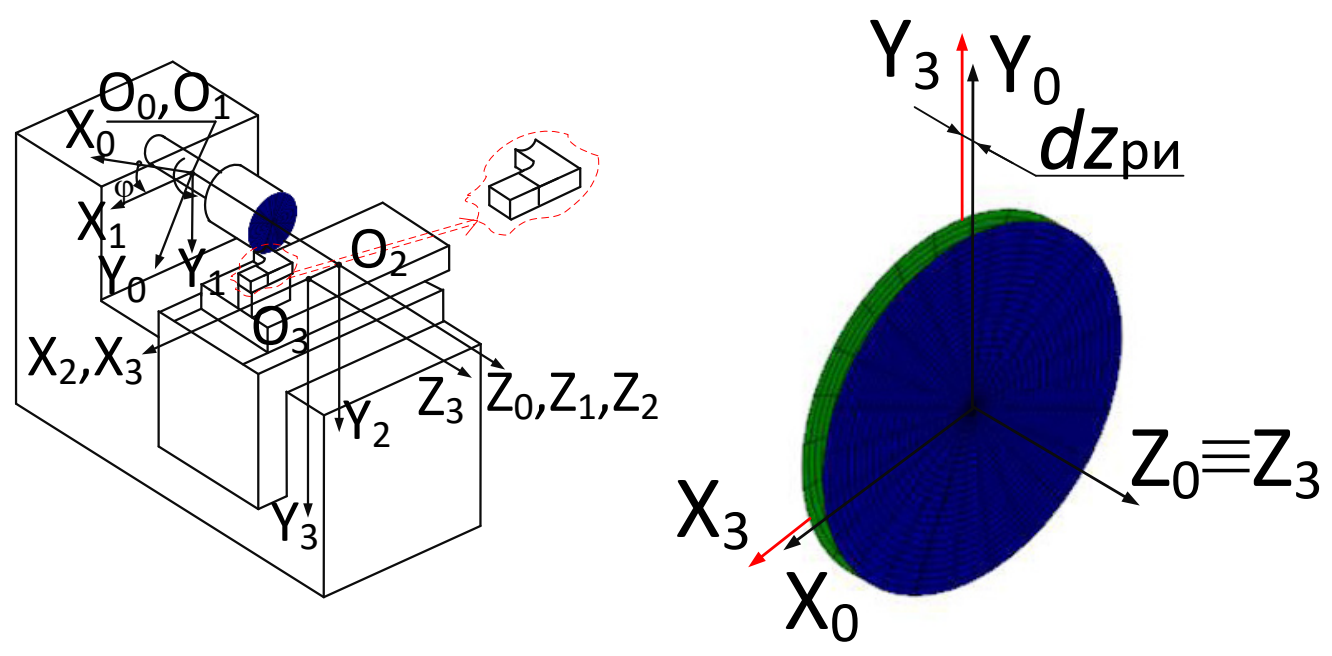

C
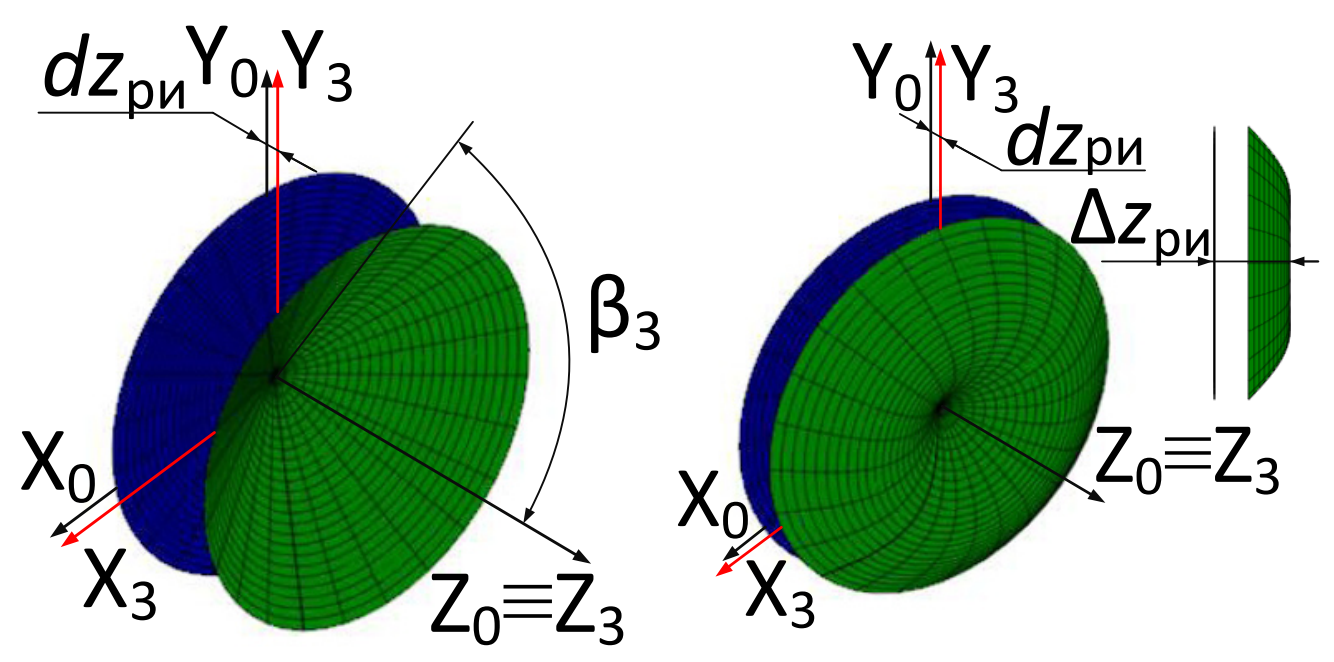

Fig. 2. Influence of MCS geometrical errors on errors of the face surface processing: a) the formbuilding system of the machine and the nominal processed surface (end face); b) formation of the real surface size error at $d z_{p u} \neq 0$; c) formation of the real surface form error at $\beta_{3} \neq 0$; d) formation of the real surface form error at $\delta z p u \neq 0$.

\section{Conclusions}

The bases of variation method for metal-cutting systems accuracy calculation systemically are stated in the presented work for the first time. The new general mathematical model of turning metal-cutting system with use of the cutting tools which can't be considered dot tools is presented. The accuracy modeling at processing cylindrical and face surfaces is performed. The results received in general are coordinated with the previous researches 
however allow considering influence of the cutting tool accuracy on processing accuracy. The further researches directions in the field of metal-cutting systems accuracy calculations when processing difficult surfaces by the shaped cutting tool and also when accounting rigidity of these systems elements and the proceeding processes are revealed.

The work is executed with financial support of RFBR (the Project № 16-38-60049).

\section{References}

1. D.N. Reshetov, V.T. Portman, Accuracy of Machine Tools (ASME Press, NY, 1988)

2. V.T. Portman, Mechanics of accuracy in engineering design of machines and robots. Volume I: Nominal functioning and geometric accuracy (ASME Press, NY, 2018)

3. A.V. Kirichek, A.G. Ivakhnenko, E.O. Ivakhnenko, A.Y. Altukhov, Geometric accuracy of the machines with strut-type structures, International journal of applied engineering research, v. 9(21), pp. 9951-9958 (2014)

4. O.V. Anikeeva, A.G. Ivakhnenko, O.Yu. Erenkov, Modeling the Influence of Geometric Errors of Turning Machine for Accuracy Machinable Surface, Procedia Engineering, v. 206, pp. 1127-1132 (2017)

5. O.V. Anikeeva, The development of the variational method for the computation of machine-tools accuracy, Fundamental and Applied Problems of Engineering and Technology, v. 5 (319), pp. 111-118 (2016)

6. A.A. Ivakhnenko, M.L. Storublev, A.G. Ivakhnenko, Tolerance Synthesis When in Operation of Process Equipment for Electroplated Coating Application, Procedia Engineering, v. 150, pp. $742-745$ (2016)

7. E. Kushnir, V.T. Portman, A. Aguilar, W. Clark, Layout evaluation at earlier stages of machine tool design: form-shaping function-based approach, International Journal of Advanced Manufacturing Technology, v. 30, pp. 3333-3346 (2016) DOI: 10.1007/s00170-016-9667-0

8. M.Q. Chen, C.H. Yang, Dynamic compensation technology of the spindle error motion of a precision lathe, Precision Engineering, v. 11, Issue 3, pp. 135-138 (1989)

9. Y. Cao, T. Liu, Xuefeng Ye, J. Yang, Lathe errors identification based on surface topography analysis after turning, Precision Engineering, v. 46, pp. 243-253 (2016)

10. V.G. Nick, Determining measurement uncertainties of feature measurements on CMMs: Proefschrift voorgedragen tot het behalen van de graad van Doctor in de Ingenieurswetenschappen, (Proefschrift voorgedragen tot het behalen van de graad van Doctor in de Ingenieurswetenschappen, KU LEUVEN, 2011)

11. A.V. Antsev, N.I. Pasko, N.V. Antseva, Assessment of wear dependence parameters in complex model of cutting tool wear, IOP Conf. Series: Materials Science and Engineering, v. 327, 042005 (2018) DOI:10.1088/1757-899X/327/4/042005 